\title{
Nail abnormalities in patients with vitiligo*
}

\author{
Ilteris Oguz Topal ${ }^{1}$ \\ Ozgur Emek Kocaturk ${ }^{1}$ \\ Mustafa Durmuscan²
}

\author{
Sule Gungor ${ }^{1}$ \\ Hatice Duman ${ }^{1}$
}

DOI: http://dx.doi.org/10.1590/abd1806-4841.20164620

\begin{abstract}
BACKGROUND: Vitiligo is an acquired pigmentary skin disorder affecting $0.1-4 \%$ of the general population. The nails may be affected in patients with an autoimmune disease such as psoriasis, and in those with alopecia areata. It has been suggested that nail abnormalities should be apparent in vitiligo patients.

Овлестіvе: We sought to document the frequency and clinical presentation of nail abnormalities in vitiligo patients compared to healthy volunteers. We also examined the correlations between nail abnormalities and various clinical parameters.

METHODs: This study included 100 vitiligo patients and 100 healthy subjects. Full medical histories were collected from the subjects, who underwent thorough general and nail examinations. All nail changes were noted. In the event of clinical suspicion of a fungal infection, additional mycological investigations were performed.

RESUlTs: Nail abnormalities were more prevalent in the patients $(78 \%)$ than in the controls $(55 \%)$ ( $p=0.001)$. Longitudinal ridging was the most common finding (42\%), followed by (in descending order): leukonychia, an absent lunula, onycholysis, nail bed pallor, onychomycosis, splinter hemorrhage and nail plate thinning. The frequency of longitudinal ridging was significantly higher in patients than in controls $(\mathrm{p}<0.001)$.

CONCLUSIONS: Nail abnormalities were more prevalent in vitiligo patients than in controls. Systematic examination of the nails in such patients is useful because nail abnormalities are frequent. However, the causes of such abnormalities require further study. Longitudinal ridging and leukonychia were the most common abnormalities observed in this study.
\end{abstract}

Keywords: Nails; Onycholysis; Vitiligo

\section{INTRODUCTION}

Nail features are important in dermatology; various abnormalities are apparent in patients with dermatological disorders, including psoriasis, lichen planus, and alopecia areata. ${ }^{1}$ Neither the frequency nor the nature of nail abnormalities in vitiligo is known.

Vitiligo is an acquired pigmentary disorder, characterized by a loss of melanocytes, resulting in the appearance of white spots, or leukoderma. Vitiligo affects $0.1-4 \%$ of the global population. ${ }^{2}$ Recent clinical studies support the notion that vitiligo is associated with autoimmune disorders, particularly Hashimoto's thyroiditis and Graves' disease; other endocrinopathies, including Addison's disease and diabetes mellitus; alopecia areata; pernicious anemia; and inflammatory bowel disease. ${ }^{3}$ Nail abnormalities are evident in $10-66 \%$ of patients with alopecia areata. ${ }^{4}$ The nails may be attacked by the same inflammatory cells that target the hair follicles of alopecia areata patients. The presumed autoimmune etiology of vitiligo, and the association between vitiligo and alopecia areata, support the hypothesis that nail abnormalities should be apparent in vitiligo patients. ${ }^{5}$

Our aim was to determine the frequencies and types of nail abnormalities in vitiligo patients compared to normal, healthy volunteers.

Received on 07.04.2015.

Approved by the Advisory Board and accepted for publication on 03.08.2015.

* Work performed at the Department of Dermatology, Okmeydani Training and Research Hospital - Istanbul, Turkey.

Financial Support: None.

Conflict of Interest: None.

Okmeydani Training and Research Hospital - Istanbul, Turkey.

Department of Biochemistry, Adana Public Health Laboratory-Adana, Turkey.

(C2016 by Anais Brasileiros de Dermatologia 


\section{METHODS}

We conducted an open, non-randomized, paired, controlled clinical study featuring intentional sampling of vitiligo patients who visited the outpatient clinic at our Dermatology Department between December 2013 and January 2015. One hundred vitiligo patients were included. For each subject, we recorded the age, sex, clinical features of the disease, and disease duration. Vitiligo was usually clinically diagnosed; if necessary, skin biopsy material was subjected to histopathological examinations. Both fingernails and toenails were analyzed, and abnormalities noted. Additional mycological investigations were performed on patients with nail abnormalities suggesting a fungal infection. Other causes of such abnormalities, including congenital and traumatic dystrophy, were eliminated, while patients with thyroid, systemic, or other autoimmune diseases, were excluded.

The control group consisted of 100 age- and sex-matched healthy volunteers without a systemic or autoimmune disease, alopecia areata, or vitiligo; no volunteer took any medication for one month prior to the study.

The following were measured in all patients and controls: levels of hemoglobin, iron, vitamin B12, folic acid, blood glucose, anti-thyroglobulin antibodies, anti-thyroidperoxidase antibodies, thyroid stimulating hormone, free tri-iodothyronine, and free thyroxine; the concentrations of anti-hepatitis B, anti-hepatitis $\mathrm{C}$, and anti-HIV antibodies; as well as the erythrocyte sedimentation rate.

This study was approved by the Ethics Committee of the Okmeydani Training and Research Hospital (Istanbul, Turkey).

Statistical comparisons featured chi-square testing and Spearman's correlation analysis. A p-value $<0.05$ was considered to reflect statistical significance.

\section{RESULTS}

In total, 51 patients (51\%) were female and $49(51 \%)$ were male; they ranged in age from 3-78 years (mean: $34.9 \pm 16.8$ years; table 1). Most patients had focal vitiligo (39\%), followed by acrofacial $(30 \%)$, generalized $(27 \%)$ and universal vitiligo $(4 \%)$. The age at onset ranged from 3-73 years. Disease duration varied from 1 month to 39 years (mean: $4.9 \pm 6.7$ years). The following nail abnormalities were present in $78(78 \%)$ patients with vitiligo: longitudinal ridging $(42 \%)$, leukonychia $(16 \%)$, an absent lunula $(13 \%)$, nail bed pallor $(5 \%)$, onycholysis $(5 \%)$, onychomycosis $(5 \%)$, splinter hemorrhage (5\%), nail plate thinning (4\%), and clubbing (4\%). Nail abnormalities were most frequent in patients with focal vitiligo (39\%), followed by acrofacial (30\%) and generalized vitiligo (27\%).
The clinical features of the nail abnormalities in the controls and patients are listed in table 2. In the controls, longitudinal ridging, leukonychia, and an absent lunula, were the most common abnormalities, followed by onycholysis and nail bed pallor.

Nail abnormalities were significantly more frequent in vitiligo patients $(78 \%)$ than in controls $(55 \%)(p=0.001)$. Similarly, longitudinal ridging (Figure 1)was significantly more common among vitiligo patients than in controls $(\mathrm{p}<0.001)$.

We found no correlation between the presence of nail abnormalities and patient age or vitiligo duration $(\mathrm{p}=0.150$ and 0.357 , respectively; Table 3).

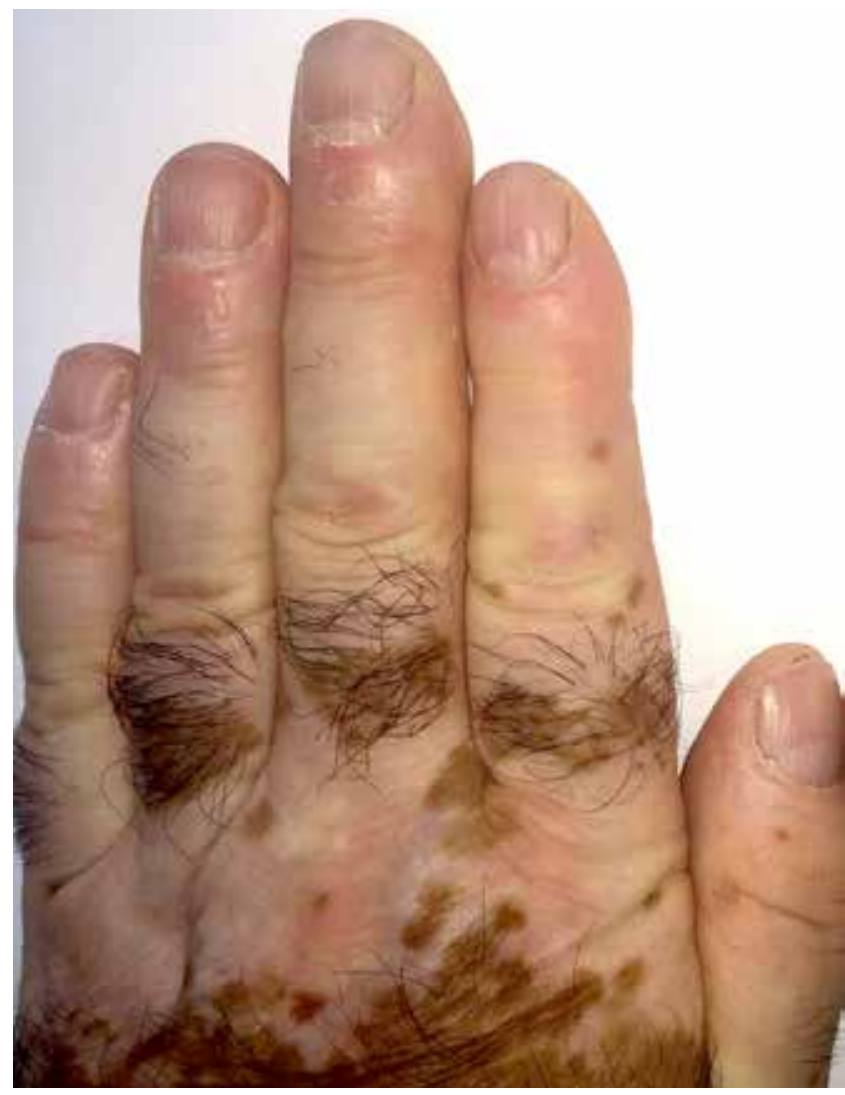

FiguRE 1: Longitudinal ridging on the nails of a vitiligo patient

TABLE 1: Demographic data of patients and controls and the frequency of nail changes in them

\begin{tabular}{|c|c|c|}
\hline & Patients & Controls \\
\hline Age (range), Mean $\pm S D$ (years) & $32(3-78), 34.9 \pm 16.8$ & $31.5(3-78), 34.8 \pm 17.2$ \\
\hline Sex (females/males) & $51 / 49$ & $50 / 50$ \\
\hline Disease duration, mean $\pm S D$ (months) & $1-39,4.9 \pm 6.7$ & \\
\hline \multicolumn{3}{|l|}{ Nail abnormalities } \\
\hline Present & $78(78 \%)$ & $55(55 \%) \mathrm{p}=0.001$ \\
\hline Absent & $22(22 \%)$ & $45(45 \%)$ \\
\hline
\end{tabular}


TABLE 2: Clinical manifestations of the nail abnormalities among patients and controls

\begin{tabular}{llll}
\hline Nail abnormality & Number of controls (\%) & Number of patients (\%) & p-value \\
\hline Longitudinal ridging & $13(13 \%)$ & $42(42 \%)$ & $<0.001^{\mathrm{a}}$ \\
Leukonychia & $13(13 \%)$ & $16(16 \%)$ & $0.688^{\mathrm{b}}$ \\
An absent lunula & $13(13 \%)$ & $13(13 \%)$ & $1.000^{\mathrm{b}}$ \\
Onycholysis & $2(2 \%)$ & $5(5 \%)$ & $0.445^{\mathrm{c}}$ \\
Nail plate pallor (pale nail) & $5(5 \%)$ & $5(5 \%)$ & $1.000^{\mathrm{b}}$ \\
Splinter hemorrhage & $1(1 \%)$ & $5(5 \%)$ & $0.212^{\mathrm{c}}$ \\
Onychomycosis & $7(7 \%)$ & $5(5 \%)$ & $0.766^{\mathrm{b}}$ \\
Nail plate thinning & 0 & $4(4 \%)$ & $0.121^{\mathrm{c}}$ \\
Clubbing & $1(1 \%)$ & $4(4 \%)$ & $0.369^{c}$ \\
Transverse ridging & $2(2 \%)$ & $3(3 \%)$ & $1.000^{c}$ \\
Nail plate thickening & 0 & $2(2 \%)$ & $0.497^{c}$ \\
Half-and-half nails & $1(1 \%)$ & $2(2 \%)$ & $1.000^{c}$ \\
Bright nail plate & $2(2 \%)$ & $2(2 \%)$ & $1.000^{c}$ \\
Longitudinal pigmentation & $1(1 \%)$ & $1(1 \%)$ & $1.000^{c}$ \\
Koilonychia & 0 & $1(1 \%)$ & $1.000^{c}$ \\
Terry nail & 0 & $1(1 \%)$ & $1.000^{c}$ \\
Thickening of the cuticle & 0 & $1(1 \%)$ & $1.000^{c}$ \\
Pitting & $1(1 \%)$ & $1(1 \%)$ & $1.000^{c}$ \\
Pterygium & $1(1 \%)$ & 0 & $1.000^{c}$
\end{tabular}

*Statistically significant $(\mathrm{p}<0.05)$

The p-values were calculated with the aid of chi-square tests (a: Pearson's chi-square test; b: Yates' [corrected] chi-square test; c: Fisher's exact chi-square test). Some patients exhibited more than one clinical manifestation.

TABLE 3: Correlation between the presence of nail abnormalities and patient age or disease duration

\begin{tabular}{lll}
\hline & \multicolumn{2}{c}{ Prevalence of nail abnormalities } \\
\hline & $\mathbf{r}$ & $\mathbf{p}$ \\
\hline Age (years) & 0.145 & 0.150 \\
Disease duration (years) & -0.093 & 0.357 \\
\hline
\end{tabular}

The p-values were calculated via Spearman's correlation analysis. r: Spearman's correlation coefficient.

\section{DISCUSSION}

Vitiligo is an acquired idiopathic disorder characterized by circumscribed, depigmented maculae and white patches. Functional melanocytes disappear from the involved skin; however, the mechanism remains unknown. ${ }^{6,7}$ Recent studies have suggested that autoimmunity plays a role in the pathogenesis of vitiligo. Further, both cellular and humoral immunity have been implicated in the development of vitiligo, as studies continue to be conducted. ${ }^{8}$ Nail abnormalities may develop in patients with autoimmune disorders, including psoriasis. Abnormalities are common (10-66\%) in patients with alopecia areata, and are also evident in $>5 \%$ of patients with autoimmune thyroiditis. Thus, the apparent associations may be real. It has been hypothesized that nail abnormalities should be apparent in vitiligo patients. ${ }^{4,9}$
Anectodal reports of nail abnormalities in vitiligo patients have appeared. Such abnormalities were first reported by Milligan $e t$ $a l .{ }^{10}$ in two vitiligo patients. Pseudo-mycotic nail dystrophy was evident and in both cases, all nails were uniformly involved (the"twenty-nails syndrome"). Later, Peloro ${ }^{11}$ described "twenty-nail dystrophy" with vitiligo in a 10-year-old female. An association between twenty-nail dystrophy (trachyonychia) and vitiligo was suggested by Barth et al. ${ }^{9}$ in 1988; these authors described eight patients with both vitiligo and nail abnormalities, including trachyonychia, pitting, onycholysis, atrophy, and pseudo-mycotic changes. Two patients had additional autoimmune disorders, and onycholysis was apparent. The detailed pathogenesis of twenty-nail dystrophy remains unknown; the condition is sometimes idiopathic, but it may occur in association with other disorders. ${ }^{11}$ Kandpur et al. described three vitiligo patients who developed twenty-nail dystrophy. ${ }^{12}$ Nail matrix biopsies revealed focal lichenoid reactions and chronic inflammatory infiltrates in the dermal papillae and around the blood vessels. It was suggested that the association between vitiligo and twenty-nail dystrophy could be explained by the autoimmune origins of both disorders. ${ }^{13}$

The literature on vitiligo patients' nails is confined to a single report on abnormalities in $79 \%$ of these patients. Longitudinal ridging and an absent lunula were significantly more common in patients than controls. The clinical form of vitiligo associated with nail involvement is principally non-segmental (71\%), followed by segmental (53.3\%). ${ }^{5}$ We studied 100 patients with non-segmental vitiligo. Nail abnormalities were evident in 39 patients (39\%) with 
focal, $30(30 \%)$ with acrofacial, and 27 (27\%) with generalized, vitiligo. Of the vitiligo patients, $78 \%$ exhibited at least one nail abnormality. Some associations were similar to those noted in the only prior study ( $\mathrm{p}=0.001$ ). The most common abnormality was longitudinal ridging, apparent in $42 \%$ of patients, followed by leukonychia (16\%) and the absence of a lunula (13\%). Nail abnormalities were not associated with abnormalities of the hands or feet.

The most common nail abnormality was longitudinal ridging ( $42 \%$ of patients; such ridging presents as a series of superficial, narrow, longitudinal, parallel striations on the nail plate). The prevalance was $2.3 \%$ at one podiatry clinic. Ridging can develop in patients with psoriasis and lichen planus, and upon trauma or aging. ${ }^{14}$ Our vitiligo patients were more frequently affected than the general podiatric populations. However, the pathogenesis remains unclear; thus, further study is needed.

The second most common nail abnormality was leukonychia $(16 \%)$. White spots (leukonychia punctata) are usually attributable to trauma, which is known to trigger vitiligo. Earlier studies of the nails of alopecia areata patients indicated that punctate leukonychia may be attributable to focal involvement of the distal matrix. ${ }^{15}$

Lunulas (the visible regions of the nail matrix ) were absent in $13 \%$ of our patients. This is the most frequent nail abnormality seen in hemodialysis patients, and has been attributed to anemia caused by chronic renal failure. ${ }^{15}$ Other common nail abnormalities in our patients were onycholysis, splinter hemorrhage, onychomycosis, and nail plate pallor.

\section{CONCLUSION}

Nail abnormalities were evident in our vitiligo patients. Vitiligo is an autoimmune disease; immune responses are abnormal, especially the roles played by $\mathrm{T}$ lymphocytes. The nails may be subject to an autoimmune attack, triggering nail abnormalities. Nail involvement was common among our patients with vitiligo. Longitudinal ridging and leukonychia were the most common abnormalities.]

\section{REFERENCES}

1. Holzberg M. Common nail disorders. Dermatol Clin. 2006;24:349-54.

2. Handa S, Dogra S. Epidemiology of childhood vitiligo: a study of 625 patients from north India. Pediatr Dermatol. 2003;20:207-10.

3. Akay BN, Bozkir M, Anadolu Y, Gullu S. Epidemiology of vitiligo, associated autoimmune diseases and audiological abnormalities: Ankara study of 80 patients in Turkey. J Eur Acad Dermatol Venereol. 2010;24:1144-50.

4. Kasumagic-Halilovic E, Prohic A. Nail changes in alopecia areata: frequency and clinical presentation. J Eur Acad Dermatol Venereol. 2009;23:240-1.

5. Anbar T, Hay RA, Abdel-Rahman AT, Moftah NH, Al-Khayyat MA.Clinical study of nail changes in vitiligo. J Cosmet Dermatol. 2013;12:67-72.

6. Di Chiacchio NG, Ferreira FR, de Alvarenga ML, Baran R. Nail trichrome vitiligo: case report and literature review. Br J Dermatol. 2013;168:668-9.

7. Ezzedine K, Lim HW, Suzuki T, Katayama I, Hamzavi I, Lan CC,et al. Revised classification/nomenclature of vitiligo and related issues: the Vitiligo Global Issues Consensus Conference. Pigment Cell Melanoma Res. 2012;25:E1-13.

8. Sandoval-Cruz M, García-Carrasco M, Sánchez-Porras R, Mendoza-Pinto C, Jiménez-Hernández M, Munguía-Realpozo P, et al. Immunopathogenesis of vitiligo. Autoimmun Rev. 2011;10:762-5.

9. Barth JH, Telfer NR, Dawber RP. Nail abnormalities and autoimmunity. J Am Acad Dermatol. 1988;18:1062-5.

10. Milligan A, Barth JH, Graham-Brown RA, Dawber RP. Pseudo-mycotic nail dystrophy and vitiligo. Clin Exp Dermatol. 1988;13:109-10.

11. Peloro TM, Pride HB. Twenty-nail dystrophy and vitiligo: a rare association. J Am Acad Dermatol. 1999;40:488-90.

12. Khandpur S, Reddy BS. An association of twenty-nail dystrophy with vitiligo.J Dermatol. 2001;28:38-42.

13. Khandpur S, Bansal A, Sharma VK, Bhatti SS, Singh MK. Twenty nail dystrophy in vitiligo. J Dermatol. 2007;34:189-92.

14. Bodman MA. Nail dystrophies. Clin Podiatr Med Surg. 2004;21:663-87.

15. Tosti A, Morelli R, Bardazzi F, Peluso AM. Prevalence of nail abnormalities in children with alopecia areata. Pediatr Dermatol. 1994;11:112-5.

\author{
MAILING ADDRESS: \\ Ilteris Oguz Topal \\ Department of Dermatology \\ Okmeydani Training and Research Hospital \\ Istanbul, Turkey \\ E-mail:drilteris@yahoo.com
}

How to cite this article: Topal IO, Gungor S, Kocaturk OE, Duman H, Durmuscan M. Nail abnormalities in patients with vitiligo. An Bras Dermatol. 2016;91(4):442-5. 